\title{
A Study of Energy Partition during Arc Initiation
}

\author{
Sotiria G. Koutoula, Igor V. Timoshkin, Senior Member, Martin D. Judd, Senior Member, \\ Scott J. MacGregor, Senior Member, Mark P. Wilson, Member Martin J. Given, Senior Member, \\ Tao Wang, and Emma I. Harrison
}

\begin{abstract}
This paper presents an analysis of energy partition among various physical processes during the formation of an electrical arc. Experimental work was conducted to accurately determine the energy delivered into the discharge. The transient voltage and current waveforms have been measured. An analytical model was developed which allows estimation of the energy partition in the discharge to be performed in order to evaluate risks associated with different energy components: thermal, kinetic-acoustic and light. Approximately $60 \%$ of the electrical energy is converted into mechanical work, subsequently contributing to the pressure rise. The results obtained will help in studies of safety considerations regarding hazards associated with plasma discharges in transient faults/sparks and during the onset of arcing faults (flash and blast hazards).
\end{abstract}

Index Terms - arc, discharge, initiation, energy, shock, flash, blast, acoustic, thermal, pressure

\section{INTRODUCTION}

$I^{N}$ $\mathrm{N}$ recent years, engineering societies have been strongly concerned with achieving a high level of safety in power systems, including minimisation of the risks associated with arcing faults (arc flash and blast) on industrial premises. Due to the dynamic behaviour of arcs, there are significant uncertainties in predicting the energy dissipation during arcing faults. Thus, selection of the most efficient protection approach poses a substantial challenge for power engineers and practitioners. Moreover, quantification tools for the hazards related to the different energy components in the arc are still under development, and further research and validation is required. A major collaborative research project between the Institute of Electrical and Electronics Engineers (IEEE) and the National Fire Protection Association (NFPA) started in 2004, to investigate experimentally the heat and thermal energy, blast pressure, sound and light during an arcing fault, in order to update the current standards

Manuscript submitted on 23 December 2015, in final form on 6 June 2016. This work was supported in part by the GSE Systems and the Faculty of Engineering and the Department of Electronic and Electrical Engineering at the University of Strathclyde.

S. G. Koutoula, I. V. Timoshkin, S. J. MacGregor, M. P. Wilson, M. J. Given, and T. Wang are with the Electronic and Electrical Engineering Department, University of Strathclyde, Glasgow, G1 1XQ, U.K. (e-mail: sotiria.koutoula@strath.ac.uk)

M. D. Judd is with the High Frequency Diagnostics \& Engineering, Glasgow, G14 0BX, U.K.

E. I. Harrison was with the GSE Systems, Glasgow, G1 1XQ, U.K. concerning arc flash safety, [1-4]. In addition, research is focused on the energy transfer mechanisms within an arcing fault and the electrical energy conversion processes, [5, 6].

The primary motivation for the research presented in this paper is to evaluate the energy partition that takes place at the very early phase of the transient arcing process. The physical processes during the initiation phase of the arc govern the evolution of the arcing fault. The estimation of different energy components at the early stages of arc development provides useful information for both qualitative and quantitative approaches to analyzing the related hazards due to the arcing fault. Improved understanding of the physical mechanisms and processes in the arc will help in identifying potential means to control its impact on personnel and power systems.

The paper consists of two main parts. The first part describes experimental work conducted to determine the electrical parameters associated with initiation of the arc discharge. Arc current and voltage waveforms were obtained and the electrical energy deposited in the arc discharge was calculated. The second part presents an analytical model, developed on the basis of hydrodynamic theory. This model allows the energy partition during the initiation phase of the arc fault to be determined. The energy components associated with thermal, acoustic and light emission processes are deduced. The pressure due to the acoustic shock wave was also determined.

\section{EXPERIMENTAL SET-UP}

The apparatus shown in Fig. 1 was used to create and study short transient arc discharges across a busbar gap, the discharges being initiated using thin conductive wires placed between the busbar and a grounded plate (a wire-guided spark gap, WGSG). An $80 \mathrm{nF}$ capacitor, $C$, is charged through a $25 \mathrm{M} \Omega$ charging resistor, $R_{c h}$, which limits the charging current and protects the HVDC power supply. A discharging resistor, $R_{s f}$, is fitted in parallel with the capacitor for safety reasons. A plasma closing switch, PCS, is inserted in series with the capacitor and serves to deposit the required energy for the short transient arc discharge across the WGSG. The inter-electrode gap of the PCS $(4 \mathrm{~mm})$ was selected to limit the breakdown voltage to $<20 \mathrm{kV}$ and the energy available in the discharge to $<10 \mathrm{~J}$.

The WGSG was connected in series with the PCS. The 
WGSG consists of a horizontal aluminium busbar used as a high-voltage electrode, and a grounded base plate with a crocodile clip for holding a $50-\mu \mathrm{m}$-diameter copper wire, the other end of this wire being attached to the busbar using a screw. A PVC frame was used to mechanically support the high-voltage electrode and the capacitor. The equivalent circuit of the arrangement is shown in Fig. 2.

Spark discharge initiation in the WGSG was achieved through the fast Joule evaporation of a copper wire, following closure of the PCS. These spark discharges were used as model electrical arcs in an investigation of the arc formation processes and energy partition during arc initiation.

Using a wire-guided discharge allows the inter-electrode distance in the WGSG to be varied from $1 \mathrm{~cm}$ to $8 \mathrm{~cm}$ for applied voltages below $20 \mathrm{kV}$, [7]. Typical gap distances between conductors in high-voltage equipment are between $1 \mathrm{~cm}$ and $15 \mathrm{~cm}$ for $1 \mathrm{kV}$ to $15 \mathrm{kV}$ ratings, thus the gaps examined in the present study were in a similar range. All experiments were conducted under normal laboratory

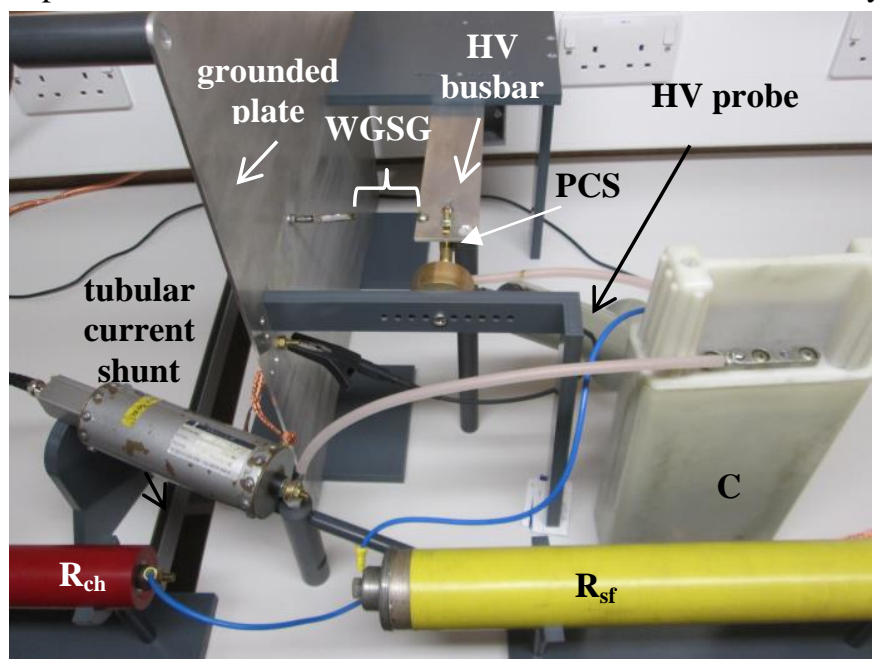

Fig. 1. The test system used in the energy partition study. C, the energystorage capacitor; PCS, the plasma closing switch; WGSG, the wire-guided spark gap; $\mathrm{R}_{\mathrm{ch}}$, the charging resistor; $\mathrm{R}_{\mathrm{sf}}$, the discharging (safety) resistor.

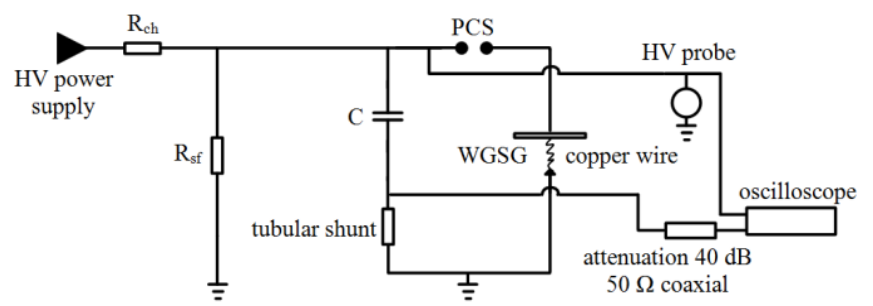

Fig. 2. Equivalent circuit of the capacitive energy-storage circuit with the PCS, the WGSG and the measurement circuitry.

conditions (an average temperature of $23{ }^{\circ} \mathrm{C}$ and an average relative air humidity of $37 \%$ ).

All waveforms were acquired using a Tektronix TDS 2024 (200 MHz, 2 GS/s) oscilloscope. A Samtech DE(CP)-01 tubular current shunt $(13.96 \mathrm{~V} / \mathrm{kA})$ was used to measure the current. A tubular shunt was chosen over other options because of its fast response and its measuring accuracy, since it is considered to react as a pure ohmic resistor that has no self-inductance. Since the use of this shunt requires interruption of the circuit conductors (the shunt needs to be electrically-inserted into the loop in series with the WGSG), efforts have been made to optimise the circuit design to provide minimum inductance.

The total inductance of the loop was calculated and found to be $\sim 700 \mathrm{nH}$, using the empirical rule of thumb $1 \mathrm{nH} / \mathrm{mm}$, [8].

The breakdown voltages were measured using either a North Star PVM-5 HV probe (1000:1, $100 \mathrm{kV}$ peak pulse) or a Tektronix P6015A HV probe (1000:1, $40 \mathrm{kV}$ peak pulse). In order to determine the electrical parameters, voltage, current and resistance of the PCS alone, the WGSG was excluded from the test system by setting a $0 \mathrm{~mm}$ gap, i.e. the WGSG electrodes were in contact, and the copper wire was absent. Typical voltage and current waveforms of the PCS are given in Fig. 3.

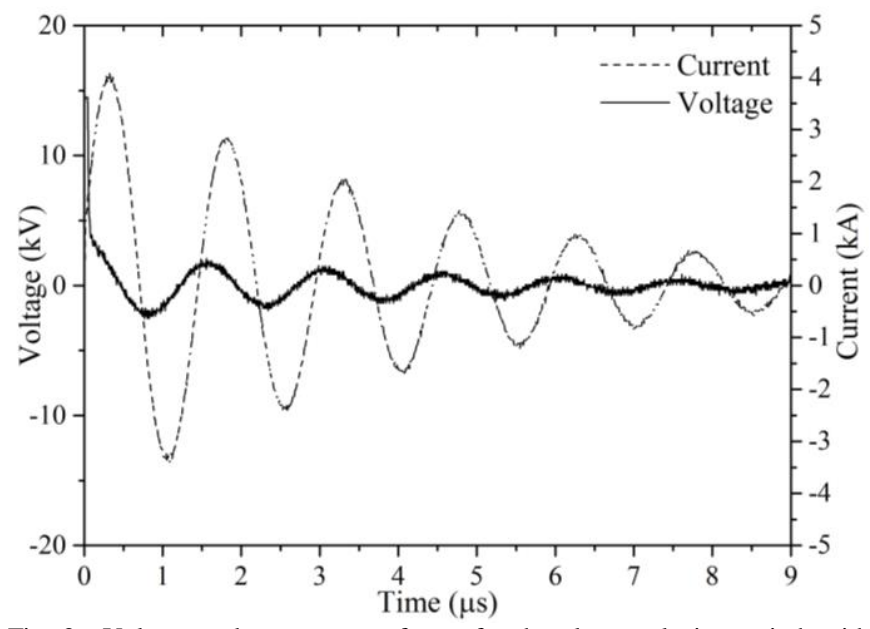

Fig. 3. Voltage and current waveforms for the plasma closing switch with inter-electrode gap of $4 \mathrm{~mm}$, acquired to calculate the energy losses in the switch.

At this point, it is necessary to introduce the term "equivalent constant resistance", which refers to the effective resistance of the circuit or element under consideration. In the present work, two equivalent constant resistances have been obtained and used in the energy partition analysis: the equivalent constant resistance of the discharge circuit with the PCS only; and the equivalent constant resistance of the discharge circuit including both gaps, the PCS and the WGSG.

The equivalent constant resistance of the circuit with the PCS only, $R_{p c s}$, consists of two components: the constant resistance of the spark discharge in this gap, $R_{\text {spark }}$, and the stray resistance of the remaining circuit, $R_{\text {stray }}: R_{p c s}=R_{\text {spark }}+$ $R_{\text {stray. }} . R_{\text {pcs }}$ was calculated following the methodology described in Section III when the inter-electrode gap in the WGSG was $0 \mathrm{~mm}$, as stated above. The average value of $R_{p c s}$ was calculated using 5 independent measurements and found to be $0.30 \Omega$ with a standard deviation value of $0.01 \Omega$.

The equivalent constant discharging circuit resistance, $R_{d}$, is the total resistance of the circuit which includes both the PCS and the WGSG. Therefore, $R_{d}$ is the sum of two equivalent constant resistances: the equivalent resistance of the circuit including the PCS, $R_{p c s}$, and the equivalent resistance of the wire-guided discharge alone, $R_{p l}: R_{d}=R_{p c s}+R_{p l}$. $R_{d}$ was calculated following the methodology described in Section III for each wire length from $1 \mathrm{~cm}$ to $8 \mathrm{~cm}$. 
After both equivalent constant resistances were obtained, $R_{p c s}$ was subtracted from the value of $R_{d}$ calculated for each individual wire length. Thus, the stray circuit resistance, $R_{\text {stray }}$, and the resistance of the PCS, $R_{\text {spark }}$, were eliminated, and the equivalent constant resistance of the plasma channel, $R_{p l}$, developed across the WGSG for each wire length was accurately defined. The energy losses in the PCS were defined in order to determine the available energy for the discharge by subtracting the PCS energy losses from the energy stored in the capacitor.

\section{EXPERIMENTAL RESULTS}

Typical voltage and current waveforms for a spark discharge created using a $3 \mathrm{~cm}$ exploding wire are presented in Fig. 4. The charging system has a time constant of $\sim 2 \mathrm{~s}$ $\left(\tau=R_{c h} C\right)$. Theoretically, the time required for the capacitor to be fully charged is $\sim 10 \mathrm{~s}$.

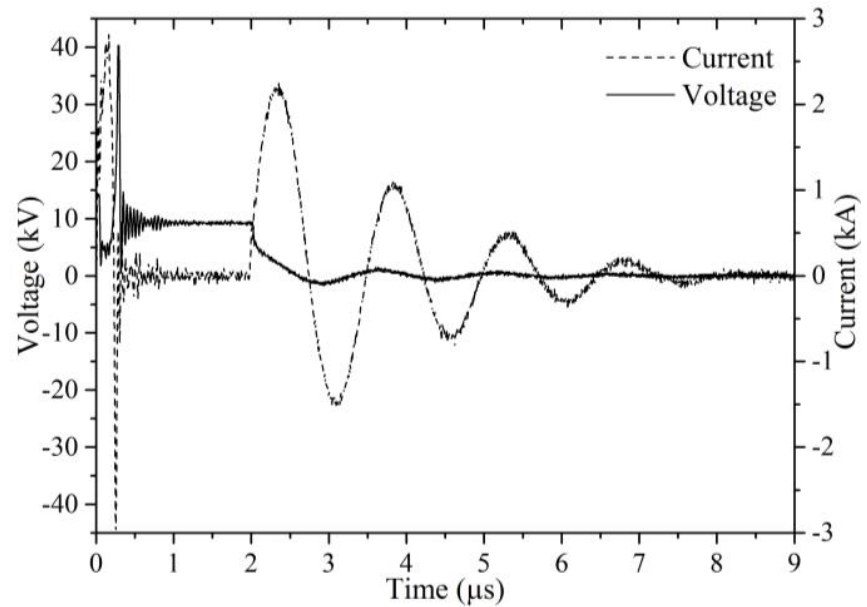

Fig. 4. Typical acquired voltage and current waveforms for a $3 \mathrm{~cm}$ wireguided spark gap with a $4 \mathrm{~mm}$ inter-electrode gap in the plasma closing switch.

Analysis of the waveforms reveals several stages of the wire-guided breakdown process. During the first $\sim 0.5 \mu \mathrm{s}$, the PCS closes and causes the rapid collapse of the voltage and increase in the current through the copper wire. At $\sim 0.5 \mu \mathrm{s}$, the wire evaporates, resulting in interruption of the initial conducting path. The WGSG opens, leading to the collapse of the current and a sharp increase in the voltage across the WGSG. The initial conducting path is interrupted for $\sim 1.5 \mu \mathrm{s}$ (this is the time interval during which the WGSG remains open, and varies stochastically between sparks), after which breakdown of the gap takes place, and the current starts to oscillate in the circuit. After breakdown the current waveform, $I(t)$, is described by an exponentially-damped sinusoidal function, (1)-(4):

$$
I(t)=I_{0} e^{-a t} \sin (\omega t)
$$

where:

$$
\begin{gathered}
I_{0}=V_{b r} C\left(a^{2} / \omega+\omega\right) \\
a=R_{d} / 2 L_{d}
\end{gathered}
$$

$$
\omega=\sqrt{1 / L_{d} C-a^{2}}
$$

$$
\begin{array}{ll}
I_{0} & : \text { current constant }[\mathrm{A}] \\
a & : \text { damping constant }[\mathrm{rad} / \mathrm{s}] \\
\omega & : \text { angular velocity of undamped oscillations }[\mathrm{rad} / \mathrm{s}] \\
V_{b r} & : \text { breakdown voltage }[\mathrm{V}] \\
C & : \text { capacitance }[\mathrm{F}] \\
L_{d} & : \text { discharging circuit inductance }[\mathrm{H}]
\end{array}
$$

Considering the arc resistance as a time-independent passive circuit element, an $R L C$ circuit with underdamped response is defined. Following the technique described in [9], the equivalent constant resistances $R_{p c s}$ and $R_{d}$ have been obtained using the experimental current waveforms, and the equivalent resistance of the plasma formed by the wire-guided discharge has been calculated: $R_{p l}=R_{d}-R_{p c s}$.

Fig. 5 presents two functional dependencies of $R_{p l}: R_{p l}$ as a function of the spark gap length, $d, R_{p l}(d)$, and $R_{p l}$ as a function of the current constant, $I_{0}, R_{p l}\left(I_{0}\right)$. The standard deviation for the $7 \mathrm{~cm}$ and $8 \mathrm{~cm}$ gaps is high because the current waveform is rapidly damped and the number of current oscillations and data points (peak current values at $\sin (\omega t)=1$ ) available for this analysis is limited. A fitting procedure in Origin Pro 9 graphing software was used to fit analytical curves to the experimental data points. These functions are displayed in (5a) and $(5 b)$ :

$$
\begin{aligned}
& R_{p l}(d)=0.08 e^{0.44 d} \\
& R_{p l}\left(I_{0}\right)=61.96 e^{-1.77 I_{0}}
\end{aligned}
$$

where $R_{p l}(d)$ and $R_{p l}\left(I_{0}\right)$ are in $\Omega, d$ is in cm, and $I_{0}$ is in kA. The analytical fitting functions (5a) and (5b) allow calculation of the plasma resistance for different arc lengths and different current constants to be made. The values of $R_{p l}$ obtained using the functional dependencies (5a) and (5b) will

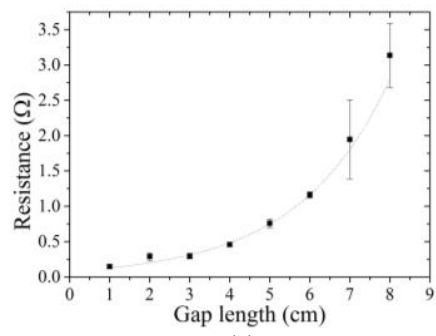

(a)

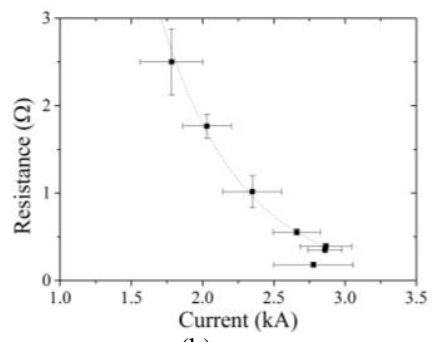

(b)
Fig. 5. Resistance, $R_{p l}$, of the spark for wire-guided spark discharges as a function of gap length, (a), and as a function of current constant, $I_{0}$, (b). The dotted lines give the best analytical fitting using (5a) and (5b) with goodnessof-fit parameters $R^{2}=0.99$ and $R^{2}=1$. The error bars represent standard deviation.

be used as input parameters in the analytical model developed in Section IV. Thus, the hydrodynamic and acoustic parameters of the transient plasma arcs can be calculated for a wide range of current waveforms.

Thus, the energy delivered into the discharge and converted 
into Joule heating can be readily calculated using the constant value of the discharge resistance, $R_{p l}$, and the current waveform. The total energy delivered in the discharge as a function of the WGSG length is given in Fig. 6.

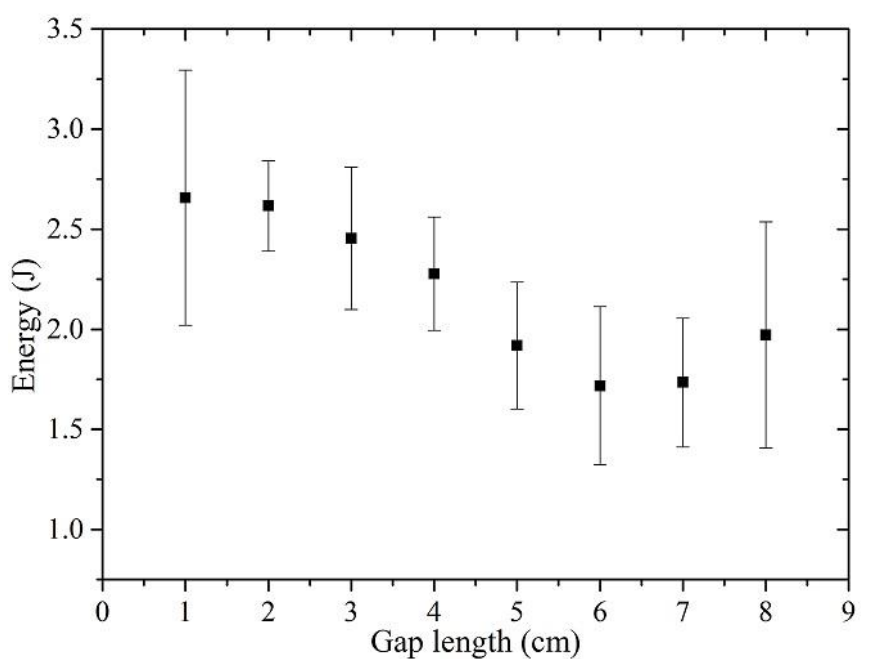

Fig. 6. Energy delivered in the discharge as a function of WGSG length. The error bars represent standard deviation.

It is evident that with an increase in the WGSG length, the energy delivered in the discharge drops because the resistance of the plasma increases, while the current drops. For the longer gaps $(7 \mathrm{~cm}$ and $8 \mathrm{~cm})$, the energy begins to show a tendency to grow. However, due to the high standard deviation, particularly at $8 \mathrm{~cm}$, it is not clear if this tendency is statistically significant.

\section{ANALYTICAL Model}

The present section is focused on estimation of energy partition into thermal energy, mechanical work and light emission. An analytical model will now be developed on the basis of hydrodynamic theory, in order to predict the energy partition that takes place during the arc initiation phase.

Previously-developed theory describes the arc channel expansion based on the concepts of hydrodynamics and considers the plasma channel as a piston that pushes the surrounding medium, establishing a shock wave $[10,11]$. Several publications report the dynamics of the arc plasma expansion, with emphasis given to the pressure and velocity fields produced in liquids, [7, 12-16]. Moreover, several studies have investigated the dynamics of arc plasma expansion in gases, $[10,11,17,18]$. Since the electrical discharge involves complex electronic and hydrodynamic processes, the development of the discharge can be described with the help of certain parameters: plasma channel resistance and radius, temperature, pressure and energy dissipation.

For the development of this model, a number of necessary assumptions were made. Atmospheric air was considered as a Newtonian fluid (non-viscous, thermally non-conducting, ideal gas) that expands only in the radial direction, [18]. The gas density, $\rho_{0}$, was considered constant at $1.2 \mathrm{~kg} / \mathrm{m}^{3}$, and the atmospheric pressure, $P_{0}$, was considered constant at $101 \mathrm{kPa}$. The plasma channel resistance was assumed to remain constant during the phenomenon development. Although a range of temperatures for the arc plasma channel in nonthermal plasmas is referred in several publications [19-21], the plasma channel temperature is considered to be constant $(\sim 7,000 \mathrm{~K})$ in the present model, representing an average plasma temperature.

The model has been developed using Braginskii's hydrodynamic energy balance equation [11], which states that the electrical energy deposited in the plasma discharge by Joule heating is converted into the internal energy of the plasma and mechanical work done by the expanding plasma. Light emission has also been taken into account in the present model. The plasma channel is considered as a blackbody radiator with emissivity factor $\sim 0.1$ [15], and its thermal radiation is described by the Stefan-Boltzmann law. The energy losses due to heat conduction are assumed negligible during this initial stage of the arc formation.

The analytical model has been built and solved using Matlab/Simulink software with solver ode45 (DormandPrince). The energy balance equation which constitutes the basis of the model is presented in (6). This equation links the pressure in the plasma channel and its volume with the electrical energy delivered to the plasma. On the left-hand side of (6), there are three terms which represent the internal energy stored in the gas, the mechanical work done by the expanding arc plasma channel, and the energy associated with light emission from the arc. These three energy components are balanced by the electrical energy deposited into the arc plasma channel, which is given by the term on the right-hand side of (6). Combining the equations of continuity and motion results in the equation for pressure in the plasma arc (7), where the assumption was made that the discharge plasma in air rapidly transforms into a spherically-expanding hot gas "ball", and the equivalent spherical topology can be used. This assumption was discussed and confirmed in [23].

$$
\begin{gathered}
\frac{P(t) V(t)}{\gamma-1}+\int_{0}^{V(t)} P(t) d V+\int_{0}^{t} 4 \pi r(t)^{2} \varepsilon \sigma T^{4} d t=\int_{0}^{t} I(t)^{2} R_{p l} d t \\
P(t)=P_{0}+\rho_{0}\left\{\frac{3}{2}\left(\frac{d r(t)}{d t}\right)^{2}+r(t) \frac{d^{2} r(t)}{d t^{2}}\right\}
\end{gathered}
$$

Since the electrical power associated with the arc channel is measured experimentally, the arc channel radius can be expressed in terms of power during the energy deposition phase of the discharge, [22]. The arc channel radius expression is given by (8), which is the result of the combination of (6) and (7).

$$
\begin{array}{r}
\frac{d^{2} r(t)}{d t^{2}}=\frac{3(\gamma-1)}{4 \pi p_{0} r(t)^{4}}\left(\int_{0}^{t} I(t)^{2} R_{p l} d t-\int_{0}^{t} 4 \pi r(t)^{2} \varepsilon \sigma T^{4} d t\right) \\
-\frac{3 \gamma}{2 r(t)}\left(\frac{d r(t)}{d t}\right)^{2}-\frac{P_{0} \gamma}{\rho_{0} r(t)}
\end{array}
$$




$$
\begin{array}{ll}
V(t) & : \text { volume of the plasma channel }\left[\mathrm{m}^{3}\right] \\
P(t) & : \text { pressure in the plasma channel }[\mathrm{Pa}] \\
\gamma & : \text { heat capacity ratio/ ratio of specific heats }(1.4 \text { for } \\
& \text { dry air at } \left.20{ }^{\circ} \mathrm{C}\right) \\
I(t) & : \text { electrical discharge current }[\mathrm{A}] \\
R_{p l} & : \text { constant resistance of the electrical discharge }[\Omega] \\
t & : \text { duration of the electrical discharge }[\mathrm{s}] \\
r(t) & : \text { plasma column/electrical discharge radius }[\mathrm{m}] \\
\varepsilon & : \text { emissivity factor } \\
\sigma & : \text { Stefan-Boltzmann constant is } 5.67 \cdot 10^{-8}\left[\mathrm{~W} / \mathrm{m}^{2} \mathrm{~K}^{4}\right] \\
T & : \text { temperature }[\mathrm{K}]
\end{array}
$$

The utilisation of (6)-(8) to simulate the temporal development of an arc plasma channel radius, and that of the energy partition, is described in the following section.

\section{A. Simulation results}

The rapid development of the arc plasma channel due to the fast energy deposition into its small volume produces a shock wave. The surrounding environment is subjected to significant stress, which depends on the medium density, due to the propagation of this shock wave, [17]. This disturbance propagates faster than the speed of sound in atmospheric air, and results in large changes in the pressure, temperature and density of the gas. In the model described in this paper, the temperature and the density of the gas medium into which the discharge takes place were assumed to be constant. The model predicts the pressure changes of the arc plasma channel and the surrounding environment in which the shock wave propagates.

The hydrodynamic properties of the air and the expansion rate of the arc plasma channel define the pressures developed in the surrounding environment, [17]. The parameters which are required for determination of the pressure are the radius, the velocity and the acceleration of the arc plasma channel. The acceleration of the expanding arc plasma channel, $d^{2} r(t) / d t^{2}$, was calculated using (8) and its velocity, $d r(t) / d t$, and the arc radius, $r(t)$, were subsequently determined. The input parameters for the model were the resistance of the spark, $R_{p l}$, obtained using (5a) and (5b), and the fitting parameters $I_{0}, \alpha$ and $\omega$, of the current waveform, obtained using (1), which were used to estimate the total electrical energy deposited into the discharge (RHS of (6)). The values of the velocity and acceleration of the arc plasma radius were then substituted into (7), to estimate the internal pressure of the arc plasma channel.

Fig. 7 presents typical waveforms obtained for the radius, velocity and internal pressure of the arc plasma channel for a $3 \mathrm{~cm}$ wire-guided discharge. At the end of the energy deposition phase, the value of the arc plasma channel radius is of the order of several $\mathrm{mm}$. Velocities of expansion of the arc of a few $\mathrm{km} / \mathrm{s}$ were obtained. Even at the end of the energy deposition stage, the velocity of the arc plasma channel is above the speed of sound. Moreover, the internal pressure developed within the arc plasma channel can reach extremely high values, of the order of tens of MPa, especially in the very early stages. During the evolution of the phenomenon, the internal pressure of the plasma channel decreases rapidly until it becomes equal to the normal atmospheric pressure when the expansion of the plasma channel ceases.

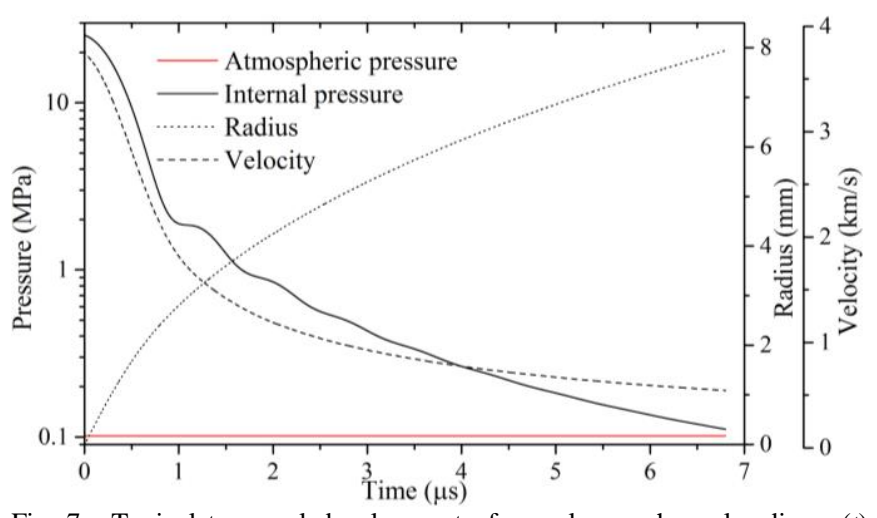

Fig. 7. Typical temporal development of arc plasma channel radius, $r(t)$, velocity, and pressure in the arc plasma channel, $P(t)$, for $3 \mathrm{~cm}$ wire-guided discharge.

Fig. 8 presents the temporal development of the mechanical work done by the expanding arc plasma channel, the internal energy stored in the gas, and the light emission. These three energy components were calculated using the expressions stated on the LHS of (6). It can be observed that, at the very beginning of the discharge, the main portion of the electrical energy is converted into the internal energy of the gas. However, the mechanical work component quickly becomes significant. During the evolution of the discharge, the electrical energy partitions mainly into the mechanical work and internal energy. Losses due to light emission are significantly smaller than the aforementioned energies.

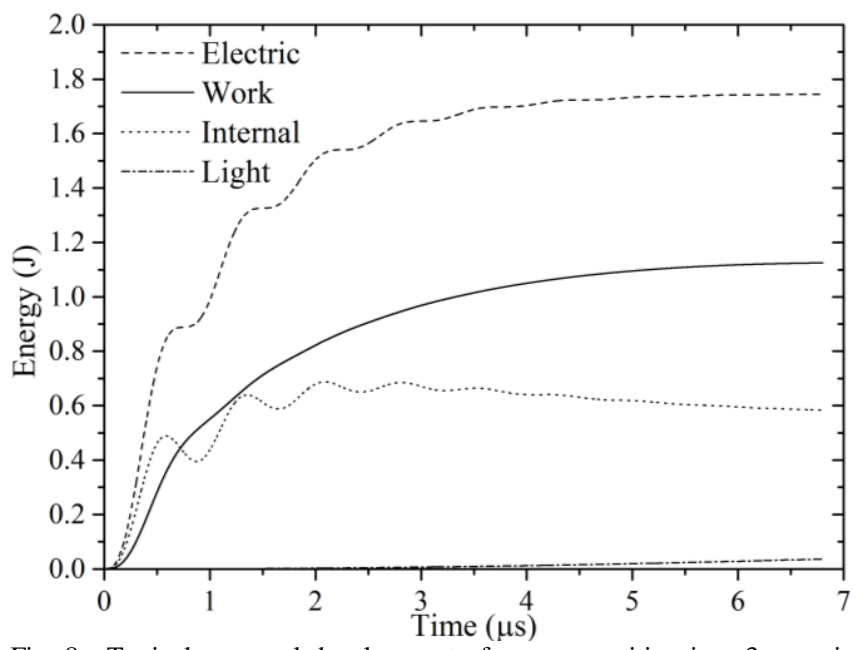

Fig. 8. Typical temporal development of energy partition in a $3 \mathrm{~cm}$ wireguided discharge.

The pressure, thermal energy and light emission with significant ultraviolet and infrared components, developed due to the mechanical work, the internal energy and the radiant energy, respectively, could potentially cause harm to personnel and damage power equipment in the vicinity of the arcing event. From a safety point of view, it is crucial to define protection boundaries for each of the potential hazards derived from the arc discharge. 
Fig. 9 shows the energy partition as a function of the total electrical energy deposited in the discharge. All energy components: internal energy, mechanical energy and light emission energy, shown in Fig. 9 represent the maximum values of these energies obtained in the simulations for each specific inter-electrode WGSG length. An example of the energy partition in the case of a $3-\mathrm{cm}$ discharge is given in Fig. 8, which corresponds to a single set of data points at $1.6 \mathrm{~J}$ in Fig. 9.

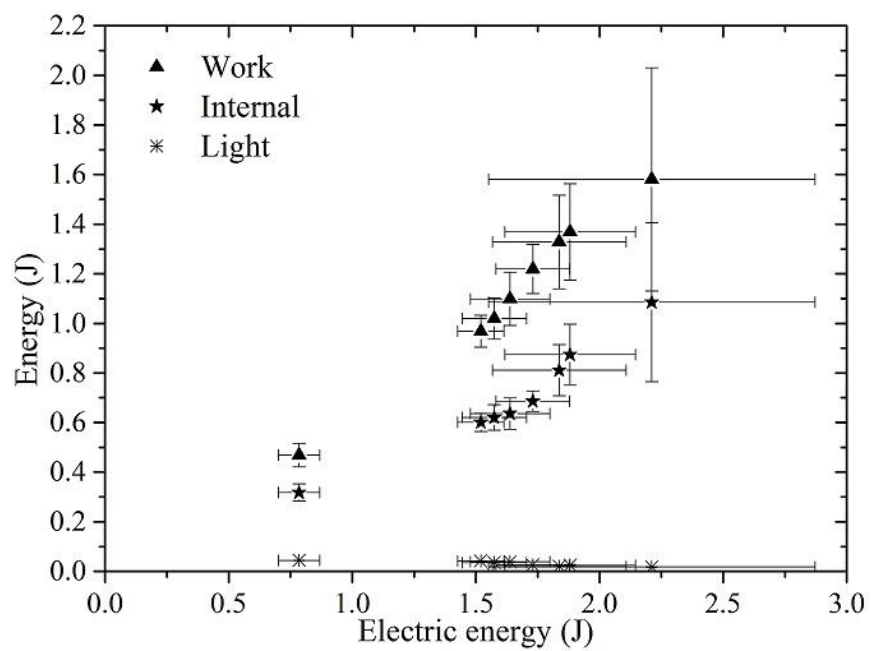

Fig. 9. Energy partition as a function of electrical energy deposited into the plasma discharge. Data points correspond to the maximum value of energy. The error bars represent standard deviation with respect to each axis.

It can be calculated that approximately $60 \%$ of the electrical energy is converted into mechanical work, subsequently contributing to the pressure rise. The rest of the electrical energy is converted into the internal energy of the gas, and afterwards into thermal energy. A portion of the electrical energy which is converted into radiant energy ( 2\%) is substantially smaller than other energy components. The model has assumed that heat conduction is not significant during the formation and expansion of the arc.

The acoustic pressure, $P_{a c}$ (the pressure in the radiated acoustic impulse), was obtained using the acoustic approximation, i.e. the pressure wave is assumed to be a compression wave that propagates in air at the speed of sound, $c$, and its measured value at a certain distance away from the plasma discharge source is given by (9), where $\tau$ is the retarded time $\tau=t-x / c$ :

$$
P_{a c}=P_{0}+\rho_{0}\left\{\frac{1}{x}\left(r(\tau)^{2} \frac{d^{2} r(\tau)}{d \tau^{2}}+2 r(\tau)\left(\frac{d r(\tau)}{d \tau}\right)^{2}\right)-\frac{r(\tau)^{4}}{2 x^{4}}\left(\frac{d r(\tau)}{d \tau}\right)^{2}\right\}
$$

Fig. 10 presents the acoustic pressure, $P_{a c}$, as a function of the distance, $x$, from the plasma discharge for all tested plasma arc lengths. It is expected from the literature that the pressure should decrease rapidly as the distance from the spark plasma discharge is increased: the pressure waveform is expected to follow a power allometric form, $P_{a c}(x) \propto x^{-2},[12,24]$. In the present model, the pressure decreases rapidly and seems to follow a hyperbolic form. The pressure levels developed are dependent on the wire-guided spark gap length and specific energy available in the discharge. However, this dependency is only evident at distances very close to the discharge. For longer distances, the pressure levels developed seem to be independent of the energy deposition. In the given experimental conditions, the pressure developed at $\sim 50 \mathrm{~cm}$ away from the discharge does not exceed $200 \mathrm{kPa}$, and the pressure is essentially equal to the normal atmospheric pressure for longer distances, $1 \mathrm{~m}$ and above.

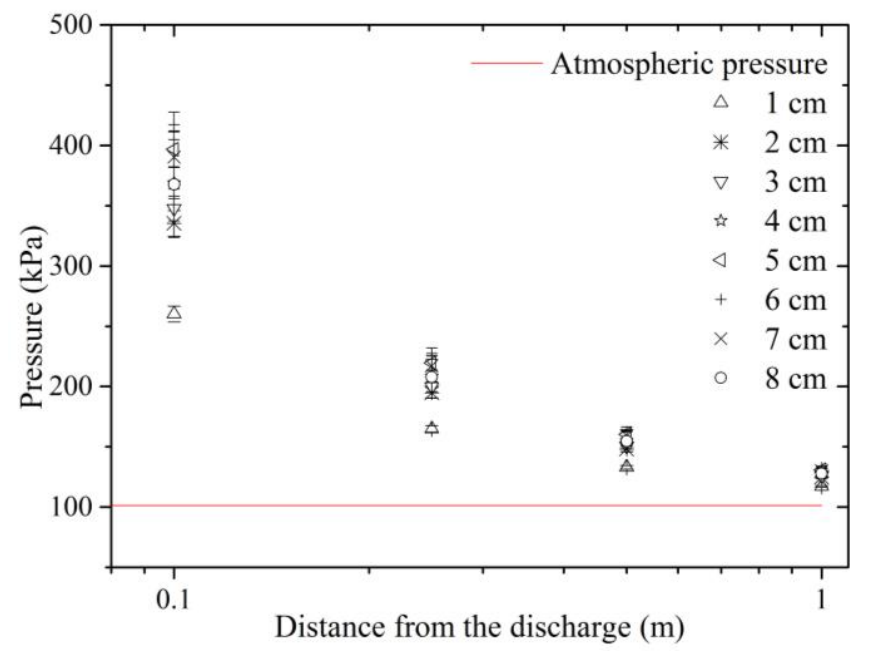

Fig. 10. Acoustic shock wave pressure as a function of distance from the discharge. The error bars represent standard deviation.

This study is focused on hazard investigation for timelimited energy deposition into the discharge. Such limited energy deposition time intervals occur during the initiation of an arc, or during transients that appear in power systems. The potential impact of longer-duration energy deposition on power systems is greater, and should be modelled. The assumptions made for this model development are unlikely to remain applicable to the simulation of a discharge in which the energy deposition phase lasts for several seconds. This is an area that requires further development.

\section{CONCLUSIONS}

Experimental results obtained from wire-guided spark discharges were presented, in order to provide information on the electrical characteristics associated with transient plasma discharges. The current waveform takes an exponentiallydamped sinusoidal form. The equivalent constant resistance was calculated for 8 different inter-electrode WGSG lengths. Thereafter, the electrical energy deposited into the discharge and converted into Joule heating for each of the gap lengths was calculated.

In addition, an analytical model was developed to estimate the energy partition among mechanical work, internal energy and radiant energy, to evaluate the risks associated with the different energy components of the discharge. It was calculated that more than half of the electrical energy was converted into mechanical work done by the expanding plasma channel radius, resulting in a subsequent pressure rise. The rest of the electrical energy was converted into internal 
energy of the gas and then into thermal energy. The radiant energy is significantly smaller than these energy components. The model has assumed that heat conduction is not significant during the formation and expansion of the arc.

Fast energy deposition into the small volume of the plasma channel results in the rapid development of the arc plasma channel, and produces a shock wave. The internal pressure developed within the arc plasma channel can reach extremely high values of the order of tens of MPa, especially in the very early times of the phenomenon. Even for this time-limited energy deposition duration, pressures 4 times higher than the atmospheric pressure are developed at $10 \mathrm{~cm}$ away from the discharge. There are aspects of the phenomenon that have not been addressed due to the assumptions made in the development of the present model. Further simulations should be performed for discharges that last longer (several seconds). In such discharges, the thermal radiation and convection are significant components of the thermal energy that should be taken into consideration in the heat transfer.

\section{ACKNOWLEDGMENT}

S.G. Koutoula is grateful for the financial support from GSE Systems Ltd, the Faculty of Engineering and the Department of Electronic and Electrical Engineering at the University of Strathclyde.

\section{REFERENCES}

[1] Guide for Performing Arc-Flash Hazard Calculations, IEEE 1584, 2002

[2] NFPA 70E Standard for Electrical Safety in the Workplace, NFPA 70E, 2012.

[3] L. Wei-Jen, T. Gammon, Z. Zhenyuan, B. Johnson, and S. Vogel, "IEEE/NFPA Collaboration on Arc Flash Phenomena Research Project," IEEE Power and Energy Magazine, vol. 10, pp. 116-123, Mar./Apr. 2012.

[4] T. Gammon, W. Lee, Z. Zhang, and B. Johnson, "Electrical Safety, Electrical Hazards, and the 2018 NFPA 70E: Time to Update Annex K?" IEEE Transactions on Industry Applications, vol. 51, pp. 2709 - 2716, Jul./Aug. 2015.

[5] D. Sweeting and A. D. Stokes, "Energy transfers within arcing faults in electrical equipment," in Proc. $8^{\text {th }}$ International Conference on Electric Fuses and their Applications (ICEFA), Clermont-Ferrand, France, Sep. 10-12, 2007, pp. 169-178.

[6] L. B. Gordon and N. Graham, "A complete electrical arc hazard classification system and its application," in Proc. IEEE IAS Electrical Safety Workshop (ESW), Louisville, KY, Jan. 26-30, 2015, pp. 1-12.

[7] I. V. Timoshkin, S. J. MacGregor, M. J. Given, and M. P. Wilson, "Plasma-acoustic sources and their practical applications," in Proc. $17^{\text {th }}$ International Conference on Gas Discharges and Their Applications (GD), Cardiff, UK, Sep. 7-12, 2008, pp. 381-384.

[8] G. D. Vendelin, A. M. Pavio, U. L. Rohde, Microwave Circuit Design Using Linear and Nonlinear Techniques, Wiley-Blackwell,2005.

[9] W. D. Greason, "Methodology to study the resistance of spark discharges," in Proc. 32 $2^{\text {nd }}$ IAS Annual Meeting Industry Applications Conference, New Orleans, LA, Oct. 5-9, 1997, pp. 1907-1913, vol.3.

[10] S. I. Drabkina, "On the theory of development of spark discharge channel," Journal of Experimental and Theoretical Physics, vol. 21, pp. 473483, 1951.

[11] S. I. Braginski, "On the theory of spark channel development," Journal of Experimental and Theoretical Physics, vol. 34, pp. 1548-1557, 1958.

[12] I. V. Timoshkin, R. A. Fouracre, M. J. Given, and S. J. MacGregor, "Hydrodynamic modelling of transient cavities in fluids generated by high voltage spark discharges," Journal of Physics D: Applied Physics, vol. 39, p. 4808, 2006.

[13] K. S. Hunter and T. L. Geers, "Pressure and velocity fields produced by an underwater explosion," The Journal of the Acoustical Society of America, vol. 115, pp. 1483-1496, 2004.

[14] S. Buogo, J. Plocek, K. Vokurka, "Efficiency of energy conversion in low-voltage spark discharges and associated bubble oscillations: Experimental results," Acta Acustica united with Acustica, vol. 95, pp. 46-59, Jan./Feb. 2009.

[15] R. M. Roberts, J. A. Cook, R. L. Rogers, A. M. Gleeson, and T. A. Griffy, "The energy partition of underwater sparks, " The Journal of the Acoustical Society of America, vol. 99, pp. 3465-3475, 1996.

[16] J. Kudelcik, M. Gutten, P. Virdzek, "Measurement of electrical parameters of breakdown in transformer oil," Przegląd Elektrotechniczny (Electrotechnical Review), vol. 87, pp. 159- 162, 2011.

[17] E. A. Martin, "Experimental Investigation of a High-Energy Density, High-Pressure Arc Plasma," Journal of Applied Physics, vol. 31, pp. 255-267, 1960.

[18] S. I. Tkachenko, D. V. Barishpoltsev, G. V. Ivanenkov, V. M. Romanova, A. E. Ter-Oganesyan, A. R. Mingaleev, T. A. Shelkovenko, and S. A. Pikuz, "Different mechanisms of shock wave generation and scenarios of second breakdown development upon electrical explosion of wires," in Proc. 16th IEEE International Pulsed Power Conference, Albuquerque, NM, Jun. 17-22 2007, pp. 877-880.

[19] C. Tendero, C. Tixier, P. Tristant, J. Desmaison, and P. Leprince, "Atmospheric pressure plasmas: A review," Spectrochimica Acta Part B: Atomic Spectroscopy, vol. 61, pp. 2-30, 2006.

[20] A. Bogaerts, E. Neyts, R. Gijbels, and J. van der Mullen, "Gas discharge plasmas and their applications," Spectrochimica Acta Part B: Atomic Spectroscopy, vol. 57, pp. 609-658, 2002.

[21] A. Fridman, Plasma chemistry, Cambridge University Press, 2008.

[22] R. J. Lee, "Ignition in solid energetic materials due to electrical discharge", Indian Head Division Naval Surface Warfare Center Report IHTR №1925, 1996.

[23] L. Hammond, "Underwater shock wave characteristics of cylindrical charges", Aeronautical and Maritime Research Laboratory Report DSTO-GD0029 (Melbourne, Australia), 1995.

[24] K. A. Naugol'nykh and N. A. Roi, Spark Discharges in Water (A Hydrodynamical Description) (translation, Foreign Technology Division, Wright-Patterson AFB, OH, 1974) (Nauka, Moscow, 1971).

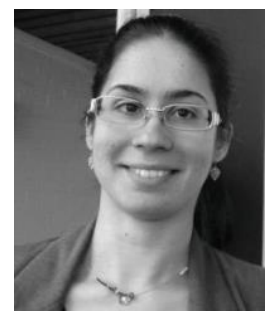

Sotiria G. Koutoula was born in Serres, Greece, in 1986. She received the M.Eng. degree in Electrical and Computer Engineering from Aristotle University of Thessaloniki (AUTh), Thessaloniki, Greece, in 2011 and is currently pursuing the Ph.D. degree in Electronic and Electrical Engineering at the University of Strathclyde, Glasgow, UK. Her research interests include electrical arc flash hazards, arc flash risk reduction and shock waves developed during arc discharges.

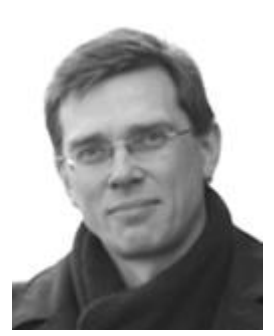

Igor V. Timoshkin (M'07-SM'14) received a degree in physics from the Moscow State University (Russia) in 1992, and the Diploma and the Ph.D. degree from the Imperial College of Science, Technology and Medicine, (London, UK) in 2001. After graduation from MSU he worked as a Researcher at Moscow State Agro-Engineering University, and then at the Institute for High Temperatures of Russian Academy of Sciences before moving to ICSTM in 1997. He joined the Department of Electronic and Electrical Engineering of the University of Strathclyde (Glasgow, UK) in 2001 where he became a Reader in 2016. His research interests include dielectric materials, electronics of plasma discharges in condensed media, gas discharges, non-thermal plasma, transient plasma in water, bio-dielectrics and effects of electromagnetic fields on biological objects. 


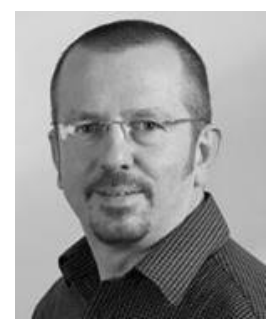

Martin D. Judd (M'02-SM'04) graduated from the University of Hull in 1985 with a BSc (Hons) first class in Electronic Engineering. He subsequently gained 8 years of industrial experience with Marconi Electronic Devices Ltd and EEV, both in Lincoln England. He received a $\mathrm{PhD}$ from the University of Strathclyde in 1996 for research into the excitation of UHF signals by partial discharges in gas insulated switchgear. Martin has worked extensively on UHF partial discharge detection and location techniques for power transformers and was latterly Professor of High Voltage Technologies and manager of the High Voltage Laboratory at the University of Strathclyde. In 2014 he founded the company High Frequency Diagnostics where he is presently Technical Director.

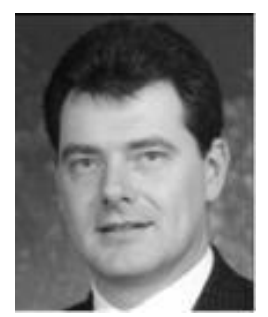

Scott J. MacGregor (M'95-SM'14) received the B.Sc. and Ph.D. degrees from the University of Strathclyde, Glasgow, U.K., in 1982 and 1986, respectively. He became a Pulsed Power Research Fellow in 1986 and a Lecturer in pulsed-power technology in 1989. In 1994, he became a Senior Lecturer, with a promotion to Reader and Professor of High Voltage Engineering, in 1999 and 2001, respectively. In 2006 and 2010 he became Head of the Department of Electronic and Electrical Engineering and Executive Dean of the Faculty of Engineering, and has been the Vice-Principal of the University of Strathclyde since 2014. His research interests include high-voltage pulse generation, high-frequency diagnostics, high-power repetitive switching, high-speed switching, electronic methods for food pasteurization and sterilization, generation of high-power ultrasound (HPU), plasma channel drilling, pulsed-plasma cleaning of pipes, and stimulation of oil wells with HPU.

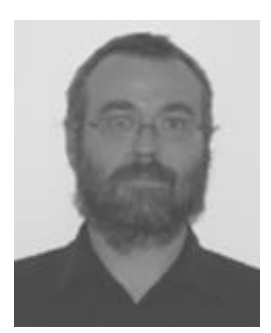

Martin J. Given (M'99-SM'11) is currently a Senior Lecturer in the Department of Electronic and Electrical Engineering at the University of Strathclyde. He received a degree in physics from the University of Sussex in 1981 and $\mathrm{PhD}$ in electronic and electrical engineering from the University of Strathclyde in 1996. His research interests include, ageing processes and condition monitoring in solid and liquid insulation systems, high speed switching and pulse power.

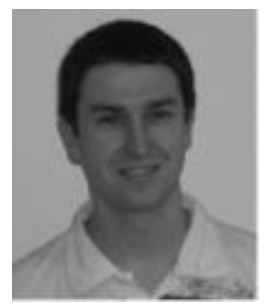

Mark P. Wilson (M'10) was born in Stranraer, Scotland, in 1982. He received the B.Eng. (with honours), M.Phil., and $\mathrm{Ph} . \mathrm{D}$. degrees in electronic and electrical engineering from the University of Strathclyde, Glasgow, U.K., in 2004, 2007, and 2011, respectively. $\mathrm{He}$ is presently working as a Teaching Associate at the University of Strathclyde, where he continues to investigate surface flashover of solids immersed in insulating oil. Mark is a member of the IEEE Nuclear and Plasma Sciences Society, from whom he received a Graduate Scholarship Award in 2011, the IEEE Dielectrics and Electrical Insulation Society, and the IET.

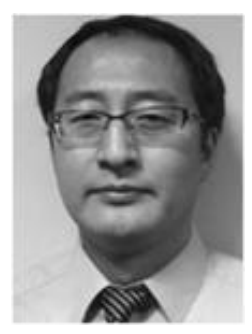

Tao Wang received the B.Eng and M.Sc degrees from Northeast China Dianli University (China) in 1993 and 1996 respectively, and the $\mathrm{Ph} . \mathrm{D}$. degree from the University of Strathclyde (Glasgow, UK) in 2005. He then joined the Newland Entech as a research fellow developing high efficiency industrial ozone generator. $\mathrm{He}$ joined the department of Electronic and Electrical Engineering of University of Strathclyde as a lecturer in 2010. His research interests include non-thermal gas discharges and their applications in gas synthesis, water disinfection and advanced oxidation process in water.

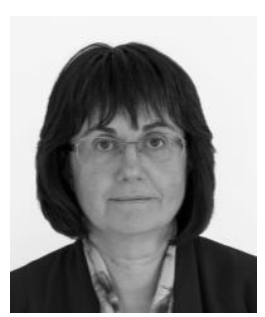

Emma I. Harrison graduated from the University of Sarajevo in 1987 with firstclass honours degree in Electrical Engineering and gained MBA from Napier University Edinburgh in 1998. She held a number of senior engineering and management roles in ABB, Rolls Royce, BAE Systems and GSE Systems where she was Managing Director of Simulation and Consultancy. 